\title{
Patient discussions of glucocorticoid-related side effects within an online community health forum
}

\author{
Authors: Arani Vivekanantham, ${ }^{A}$ Maksim Belousov, ${ }^{B}$ Lamiece Hassan, ${ }^{B}$ Goran Nenadic ${ }^{B}$ and Will Dixon ${ }^{B}$
}

\section{Introduction}

Social media websites are an important, largely untapped source of data about patients' experience of living with disease and its treatment. This includes information on drugs such as the occurrence, nature and impact of side effects. However, there are few published studies reporting drug safety profiles using such data.

Health Unlocked (HU), Europe's largest social media network for health that supports patients and healthcare providers, hosts over 200 communities including the UK's National Rheumatoid Arthritis Society (NRAS). Using the example of glucocorticoid (GC) therapy, this study aims to explore the potential of HU posts in providing information about the occurrence and nature of drug side effects.

\section{Objectives}

$>$ Evaluate the accuracy of a computerised system for automated suspected adverse drug reaction (SADR) detection from posts from $\mathrm{HU}$ compared with human annotation.

> Explore themes of discussion about GC-related adverse drug reactions (ADRs) within posts from $\mathrm{HU}$.

\section{Methods}

HU provided a dataset of deidentified posts from the NRAS community from December 2015 to December 2016. Posts mentioning GCs were processed by automated natural language processing software, which identified the drug and health issues, mapped them to the Medical Dictionary for Regulatory Activities (MedDRA) and categorised as an sADR or not. A sample $(n=50)$ of SADR posts were randomly selected and manually reviewed to determine whether they were true ADRs. Additionally, a sample $(n=50)$ of the posts that included GC and were labelled as having a health issue, but not thought to have an ADR, were also assessed for true ADRs.

Posts identified as containing GC ADRs from manual analysis were reviewed to identify themes.

Authors: AManchester University NHS Foundation Trust;

${ }^{\mathrm{B}}$ Manchester University

\section{Results}

Of the 35,904 posts from 1,998 users, 2,409 posts mentioned GCs, of which 324 posts were identified as containing information representing an SADR.

After manual review of the 50 sampled sADRs, only $36 \%(18 / 50)$ of these posts contained a true ADR. Of the 50 sampled posts that included a mention of GCs and a health issue but were not an SADR, 28\% (14/50) were found to contain true ADRs.

Thematic analysis of the 32 posts containing true GC ADRs found that the most frequently mentioned ADRs were fractures $(n=6)$, infection $(n=5)$, headaches $(n=3)$ and weight gain $(n=3)$. Posts included rich descriptions about the nature of side effects ('my weight tripled in size with steroids'). This included experiences of how side effects changed with time ("huge mood swings settled after a while'). Users also described how ADRs impacted on their quality of life ('with steroid-induced diabetes, I lost a stone in 3 days, it was grim'), and their value judgements about the importance of side effects ('my taste buds are making everything taste strange, either salty, metallic, or plain awful ... but I cope with it, as hardly any pain with steroids'). Posts also described frustrations about how well informed they were about side effects ('I had two eye ops for cataracts, no one told me steroids caused cataracts'). Within posts where ADRs were discussed, patients also commented on the benefits of treatment ('my pain subsided with steroids') and the difficult balance between benefits and harms ('wonderful to not feel like I had rheumatoid arthritis in the first month of having [pred], but now I have more acne then when I was a teenager').

\section{Conclusions}

Current machine learning models for ADR detection in social media still need further improvements to identify sADRs in health forum data. Nonetheless, manual review shows there are important themes relating to patients' experiences and perceptions of using GC that may not be obtained using traditional methods such as analysis of health records or spontaneous pharmacovigilance. With improved automated ADR detection, this rich data source may be useful to identify the ADRs most important to patients and the impact on quality of life. 